\title{
Syncategorematic Conjunction and Structured Meanings
}

\author{
Yoad Winter \\ OTS, Utrecht University
}

\section{Introduction: The Boolean Assumption and the Universal of Coordination}

One elegant and linguistically powerful guideline for a semantic theory of coordination will be referred to here as the Boolean assumption. According to this assumption conjunctive coordinators like and are described using the cross-categorial Boolean operator of Generalized Conjunction (GC). Symmetrically, disjunctive coordinators like or are modeled by Generalized Disjunction (GD). A common type theoretical definition of the operators GC and GD goes as follows:

Definition: An extensional type $\tau$ is called $t$-reducible iff $\tau=t$ or $\tau=\tau_{1} \tau_{2}$, where $\tau_{1}$ is any type and $\tau_{2}$ is a $t$-reducible type. For every $t$-reducible type $\tau$ :

$$
\begin{aligned}
& \Pi_{\tau(\tau \tau)} \stackrel{\text { def }}{=} \begin{cases}\lambda X_{t} \cdot \lambda Y_{t} \cdot X \wedge Y \\
\lambda X_{\tau} \cdot \lambda Y_{\tau} \cdot \lambda Z_{\tau_{1}} \cdot X(Z) \sqcap Y(Z) & \tau=t \\
\tau=\tau_{1} \tau_{2}\end{cases} \\
& \sqcup_{\tau(\tau \tau)} \stackrel{\text { def }}{=} \begin{cases}\lambda X_{t} \cdot \lambda Y_{t} \cdot X \vee Y \\
\lambda X_{\tau} \cdot \lambda Y_{\tau} \cdot \lambda Z_{\lambda_{1}} \cdot X(Z) \sqcup Y(Z) & \tau=t\end{cases}
\end{aligned}
$$

Modeltheoretically the operators $\Pi$ and $\sqcup$ can be shown to be indirectly representing the simple operations of set intersection and set union.

The Boolean assumption is then a linguistic assumption about the lexical

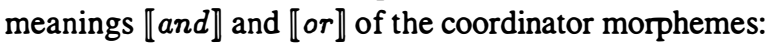

The Boolean Assumption: $\llbracket$ and $\rrbracket=\Pi$, $\llbracket$ or $\rrbracket=\sqcup .{ }^{1}$

Different variants of this assumption were profitably followed in the Boolean semantics of Keenan \& Faltz(1985) and in many other works. In Winter(1994) it was shown that also cases of collective predication (e.g. Mary and John met) can be treated using the Boolean assumption without stipulative lexical ambiguities.

Yet, in this paper it is argued that the Boolean assumption is too strong in one point: the lexical connection it makes between conjunctive coordinators like and and the Boolean operator of conjunction. It will be shown that some systematic empirical distinctions between conjunctive and disjunctive coordinations are hard to be accounted for if we follow the Boolean assumption as stated above. Data will be considered from cross-linguistic phenomena of morphologically null conjunctive coordinators, "wide scope" interpretation of conjunctions and modifiers sensitive to the internal structure of the coordination. The claim that these phenomena do not appear with disjunctive coordinations will be established, which is shown to be a major challenge for the Boolean assumption. It is argued that the data can be explained if the assumption is revised by assuming that Boolean conjunction is a syncategorematic semantic ${ }^{2}$ process in all natural languages. Conjunctive morphemes 
like and are better treated as lacking any denotational contribution to meaning. By contrast, disjunctive morphemes are standardly treated categorematically, denoted by logical disjunction as in the Boolean assumption. This distinction between conjunctive and disjunctive coordinations expresses a semantic universal about natural languages that will be referred to as the universal of coordination.

The semantic mechanism proposed for the interpretation of conjunctive coordinations involves a product logic where they are basically treated as tuples. This is a very restricted implementation of the theory of Structured Meanings in Cresswell(1985). It accounts for "wide scope" interpretations of conjunctions as well as for the possibility to modify them in a "structure sensitive" way.

\section{Distinctions between conjunctive and disjunctive coordinations}

\subsection{Zero morphology conjunction}

In many languages coordination may lack an overt coordinator. The conjuncts are juxtaposed without any material inbetween. Payne(1985) stresses that the interpretation of the coordination in these cases is systematically conjunctive. Payne distinguishes between obligatory and optional zero strategies. The obligatory strategy appears in the Samoyed languages (spoken in north ex-USSR) and in Pacoh (spoken in Mon-Khmer, Vietnam, see (1)). In these languages there is no con junctive morpheme and the only way to express conjunction is by juxtaposition of the conjuncts. In the optional zero strategy a conjunctive morpheme exists but can be omitted. Languages with this strategy include Turkish (see (2)), Tatar, and Vietnamese.

(1) do [Vp chǒ tốq cayâq chǒ tốq apây] (from Payne(1985:26)) she return to husband return to grandmother

"She returns to (her) husband and returns to (her) grandmother"

(2) [NP sen, ben, ve / $\varnothing$ kardeçin ] ${ }^{3}$ (from Payne(1985:25))

you, I and / $\varnothing$ brother-your

"you, I and your brother"

Payne notes that no language was found to have an obligatory zero strategy with respect to disjunction (p.39) and concludes: "On the whole, it is rare to find anything unusual in disjunction"(p.40). A related fact is the trivial observation that consequent sentences in discourse normally convey propositional conjunction. For example, the discourse in (3a) is logically equivalent to the sentential conjunction in (3b). A similar case is parataxis ${ }^{4}$ as in the Hebrew sentence (4).

(3) a. John is talking. Mary is walking. b. John is talking and Mary is walking.

(4) imi xaya, ani, axi, hukarnu kenexey cahal. (from Ha'aretz, 28 April 1995) mother-1:sg chaya, I, brother-1:sg, recognized-past-1:pl as-disabled-pl IDF "My mother Chaya, I, my brother, were recognized as disabled (because of) IDF service"

Certain languages probably lack coordination as familiar to English speakers. Two such cases are Maricopa (see Gil(1991)) and Dyribal (see Dixon(1972)). The Maricopa sentence (5) might seem to involve a zero disjunction strategy: 
(5) Johnš Billš v?aawuumšaa.

John-nom Bill-nom 3-come-pl-fut-infer

"John or Bill will come" (from Gil(1991:102))

However, Gil notes that if the suf fix šaa on the verb in (5) is omitted the interpretation of the sentence becomes conjunctive. This particle indicates uncertainty like the auxiliary may in English. A similar point is shown in Dixon(1972:363, example (693)) with the Dyribal particle yamba ("perhaps, might be"). A related phenomenon in English has to do again with juxtaposed sentences:

(6) Who's coming to the party? Mary is coming, John is coming... I don't know. Here too, the apparent zero disjunction appears with an overt modal expression. Concluding, a generalization adequate to the facts described above seems to be:

$\left(\mathbf{G}_{1}\right)$ Many languages allow various forms of zero conjunction. Zero disjunction is very rare and appears only with some overt expression indicating uncertainty.

\section{2 "Wide scope" coordination}

A phenomenon that has important implications for theories of coordination and quantification is one that is sometimes referred to as "the scope of coordination". I will use this term in a descriptive and theoretically neutral sense:

Terminology: A sentence that contains a structure $\left[\mathrm{X}_{1} \operatorname{coor} \mathrm{X}_{2}\right] \mathrm{Y}$ has a wide scope reading for the coordination iff it has a reading paraphrased using the structure [ $\left.\mathrm{X}_{1} \mathrm{Y}\right]$ coor $\left[\mathrm{X}_{2} \mathrm{Y}\right]$. (symmetrically for structures $\mathrm{Y}\left[\mathrm{X}_{1} \operatorname{coor} \mathrm{X}_{2}\right]$ )

A central empirical claim of this paper is that while "wide scope"(WS) conjunction is a rather common phenomenon in English, WS disjunction appears only in certain restricted cases. Consider first the sentence:

(7) John sold and bought a car. (following Hendriks(1993:52))
a. NS: $\exists x\left(\operatorname{car}^{\prime}(x) \wedge \operatorname{sell}^{\prime}\left(\mathrm{j}^{\prime}, x\right) \wedge \operatorname{buy}^{\prime}\left(\mathrm{j}^{\prime}, x\right)\right)$
b. WS: $\exists x\left(\operatorname{car}^{\prime}(x) \wedge \operatorname{sell}^{\prime}\left(\mathbf{j}^{\prime}, x\right)\right) \wedge \exists x\left(\operatorname{car}^{\prime}(x) \wedge \operatorname{buy}^{\prime}\left(\mathbf{j}^{\prime}, x\right)\right)$

The prominent reading of (7) is not the narrow scope coordination reading in (7a) which requires one car both sold and bought, but rather the WS reading in (7b), paraphrased as "John sold a car and bought a car". To see this consider a situation in which John sold his old Ford and bought a new BMW. This is a situation distinguishing the NS from the WS reading, henceforth a critical situation (CS). In this situation (7a) is contingent whereas (7b) is true. (7) can be used truthfully in this situation, so the WS reading (7b) is attested. ${ }^{5}$ As far as conjunction is concerned, this fact agrees with the claim in Hendriks(1993:52-53) about WS coordination in this construction. However, let us consider also disjunctions. It would be useless to contrast (7) with a minimally different sentence like John sold or bought a car where the NS and WS readings are equivalent. But consider:

(8) John sold or bought every house in this neighbourhood.
a. NS: $\forall x\left(\operatorname{house}^{\prime}(x) \rightarrow\left(\operatorname{sell}^{\prime}\left(\mathrm{j}^{\prime}, x\right) \vee\right.\right.$ buy $\left.\left.^{\prime}\left(\mathrm{j}^{\prime}, x\right)\right)\right)$
b. WS: $\forall x\left(\operatorname{house}^{\prime}(x) \rightarrow \operatorname{sell}^{\prime}\left(\mathrm{j}^{\prime}, x\right)\right) \vee \forall x\left(\operatorname{house}^{\prime}(x) \rightarrow \operatorname{buy}^{\prime}\left(\mathbf{j}^{\prime}, x\right)\right)$ 
Consider a CS in which the houses are A,B,C and D. John sold houses A and B and bought houses $C$ and D. According to (8a), (8) should be true. According to (8b) the sentence should be false. It seems that (8a) gives the correct prediction: it is hard to think of this situation as falsifiying (8). Thus, conjunctions as in (7) exhibit a WS reading whereas disjunctions of the same structure as in (8) do not. However, there are two weaknesses to this choice of examples: (i) (7) and (8) are not minimally different - one might suspect that the lack of the WS reading in (8) has to do with the replacement of the indefinite NP by a universal. (ii) The WS reading (8b) entails the NS (8a). It is sometimes hard to show that a logically stronger reading than some standard one exists because there might be a tendency of speakers to prefer a true reading to a false one. A speaker who hears (8) in a CS might consider (8) to mean ( $8 a$ ), just because ( $8 a$ ) is true and the aclaimed WS reading ( $8 b)$ is false.

Therefore, we are to look for a better choice of examples. For methodological reasons, let us restrict ourselves to sentences with only one coordination and one existential or universal quantification in the representation. The entailements in (9)-(12) are the relevant ones. The left side is the logical representation for the standard NS readings. The right side is the representation for the "WS" reading.

$$
\begin{aligned}
& \text { a. } \operatorname{every}(A \cap B, C) \Leftarrow \operatorname{every}(A, C) \wedge \operatorname{every}(B, C) \\
& \text { b. } \operatorname{every}(A \cup B, C) \Rightarrow \operatorname{every}(A, C) \vee \operatorname{every}(B, C) \\
& \text { a. } \operatorname{every}(A, B \cap C) \Leftrightarrow \operatorname{every}(A, B) \wedge \operatorname{every}(A, C) \\
& \text { b. } \operatorname{every}(A, B \cup C) \Leftarrow \operatorname{every}(A, B) \vee \operatorname{every}(A, C) \\
& \text { a. } \operatorname{some}(A \cap B, C) \Rightarrow \operatorname{some}(A, C) \wedge \operatorname{some}(B, C) \\
& \text { b. } \operatorname{some}(A \cup B, C) \Leftrightarrow \operatorname{some}(A, C) \vee \operatorname{some}(B, C) \\
& \text { a. } \operatorname{some}(A, B \cap C) \Rightarrow \operatorname{some}(A, B) \wedge \operatorname{some}(A, C) \\
& \text { b. } \operatorname{some}(A, B \cup C) \Leftrightarrow \operatorname{some}(A, B) \vee \operatorname{some}(A, C)
\end{aligned}
$$

The non-equivalences are (9a-b), (10b), (11a) and (12a). Thus, coordination scope should be in principle visible in the cases: and/or in the restriction of a universal, or in the scope of a universal, and in the restriction/scope of an existential. Examples (7) and (8) above contain a coordination in the scope of the existential/universal quantifier. A more contrastive test is when coordination appears in the restriction of a universal quantifier. From (9), in this situation both and and or can potentially exhibit a WS reading. Moreover, the WS or reading, if appears, should be entailed by the NS reading (cf. (9b)) and therefore should be easily attested. The minimally different sentences (13) and (14) examplify the relevant test:

(13) John likes every boy and girl in the class.

a. WS: John likes every boy in the class and likes every girl in the class.

b. CS: There is a child in the class that John doesn't like. Judgement: false, $\rightarrow$ WS attested 
(14) John likes every boy or girl in the class.

a. WS: John likes every boy in the class or likes every girl in the class.

b. CS: John hates a boy in the class.

Judgement: false, $\rightarrow$ WS unattested

The WS paraphrase (13a) for (13) is attested in a CS like (13b) where the sentence is false. This WS was often noticed in the literature (e.g. Bergmann(1982), Partee \& Rooth(1983)). By contrast, observed by Bergmann, (14) is false in a CS like (14b), so the WS reading in (14a) is unattested. A similar contrast appears in (15)-(16):

(15) Mozart is easy to play for every pianist over 60 and below 20 years old.

(16) Every person over 60 or below 20 years old is allowed to smoke here.

In (15) the prominent reading is the contingent WS and not the vacuously true NS with the empty common noun set. For (16), consider a CS in which John is 65 years old and not allowed to smoke. (16) is intuitively false like the NS reading, which means the contingent WS or is unattested. Another example for the lack of WS disjunction, by contrast to WS conjunction are the following sentences:

(17) (A woman discovered Radium but) a man invented the electric light bulb and developed the theory of Relativity. (following Hendriks(1993:53))

(18) (? The girls didn't all do equally well in the exam but) every boy failed or got an "A". (following Partee \& Rooth(1983))

This evidence is of the weaker type, similar to the contrast (7)-(8), but it strengthens the observation on WS conjunction, in distinction to WS disjunction.

However, WS or does seem to appear in examples like:

(19) John is looking for a cat or a dog. (following Rooth \& Partee(1982))

In a situation where John is looking for a cat (de dicto: any cat, not a specific one) but not for a dog it seems that (19) can be interpreted as true, which attests a WS reading for the coordination. Namely: "John is looking for a cat or looking for a dog, but I don't know which of the two kinds of pets is he looking for." More will be said about this type of examples in section 4. In the meantime, a bibliographical note is in place: although many works deal with WS disjunction in intensional contexts as in (19) (a non exhaustive list: Rooth \& Partee(1982), Larson(1985), Hendriks(1993), Emms(1993), Carpenter(1994), Moltmann(1992)), neither of these studies provides evidence for WS or in extensional contexts as in (7)-(8), (13)-(18). The fact that WS or is not attested in these cases calls for an explanation.

The distinctions found between the scope of conjunction and disjunction are summarized in the following generalization:

$\left(\mathbf{G}_{2}\right)$ WS and is attested for many different configurations in English. WS or is attested only in the presence of some intensional operator.

An empirical caveat: Even without getting into subtleties of plurals and complex syntactic constructions it should be noted that with the indefinite article WS interpretation of common noun conjunction is hard, if not impossible, whereas NS is easy to get. Compare (20a) and (20b). I will not address this question here. ${ }^{6}$ 
(20) a. ? John likes some man and woman.

b. John likes some of ficer and gentleman.

\section{3 "Structure sensitive" adverbials - altemately, respectively}

The semantic operation of certain adverbials in English is another challenge for the Boolean assumption about conjunctive coordinations. One problem with the adverb alternately is discussed in Lasersohn(1992). For example, consider the sentences:

(21) a. The cable runs alternately above ground and below ground.

b. John was alternately hot and cold. (from Lasersohn(1992))

Lasersohn shows that it is hard to get the correct interpretation of such sentences if we traditionally apply GC to some standard denotation of the conjuncts. Roughly, in (21b) if the conjunction is analyzed using the logical expression hot ${ }^{\prime} \sqcap$ cold $^{\prime}$, when hot' $^{\prime}$ and cold' are temporal predicates (functions from points of time to sets), then it denotes for every point of time the set of entities which are both hot and cold at the same time. Reasonably, this is always the empty set, which makes compositional modification using alternately impossible. Intuitively, the adverbial "needs access" to the separate denotations of the conjuncts to produce the semantics of alternation. However, such problems do not appear with or: the adverbial alternately cannot modify a disjunctive coordination. Contrast (21a-b) with:

(22) a. * The cable runs alternately above ground or below ground.

b. * John was alternately hot or cold.

A similar point holds for the adverbial respectively: it seems impossible to analyze (23) with a GC denotation for and, but disjunctions as in (24) are not licensed.

(23) Mary and Sue are tall and short respectively.

(24) * Mary and Sue are tall or short respectively.

This leads to the following generalization:

$\left(\mathbf{G}_{3}\right)$ altemately and respectively are licensed with and coordinations, not with or.

\section{Analysis}

The facts summarized in $\left(\mathbf{G}_{1}\right)-\left(\mathbf{G}_{3}\right)$ are a challenge to the Boolean assumption: if conjunctive and disjunctive coordinations are symmetrically treated using the Boolean operators what can be the origin for the systematic distinctions between them? An additional technical problem is posed by the facts in $\left(\mathbf{G}_{2}\right)$ and $\left(\mathbf{G}_{3}\right)$ : the straightforward semantics for conjunctive morphemes cannot account for WS effects and "structure sensitive" modification without further mechanisms.

The proposed conceptual change in the Boolean assumption is the following: we hypothesize that in all natural languages the semantics of conjunctive coordinations is not derived by any lexical semantic value for the (nullovert) coordinator. GC is to be used as a basic derivational semantic process in human languages. ${ }^{7} \mathrm{GD}$ standardly denotes morphemes like or. I refer to this hypothesis as: 
The Universal of Coordination: Conjunction is syncategorematic. Disjunction is categorematic.

The implementation relies on this universal. It is proposed that conjunctive coordinations are represented using Structured Meanings of the conjuncts denotations. This is the axiom of product introduction: from any two juxtaposed denotations $X, Y$ of types $\tau, \sigma$ we can derive a tuple (= a structured meaning) $\langle X, Y\rangle$ of the product type $\tau \bullet \sigma$. It is a very restricted implementation of Cresswell(1985)'s theory of mental attitudes. Conjunctive coordinations are treated as derivationally ambiguous between two readings: (i) An "intersective" reading, obtained by free application of GC to the conjunct denotations. (ii) A "product" reading, obtained by the axiom of product introduction. The first possibility maintains the similarity between conjunction and disjunction. The second is responsible for WS and "structure sensitivity" effects, special to conjunctive coordinations. Let us substantiate these ideas, dealing with the problems mentioned in section 2 .

\subsection{Zero morphology conjunction}

It should be noticed that the challenge $\left(\mathbf{G}_{1}\right)$ poses to the Boolean assumption is not a descriptive one. We can stipulate in cases of zero conjunction a null element conveying the meaning of GC. But the main question remains unanswered: why can only conjunctive coordinators be morphologically null and not disjunctive ones? The universal of coordination provides an answer: a null element is not assumed because the semantic operation of GC is null anyway- it is in the "hard core" of natural language grammar(s). There exist languages with zero conjunction because morphemes like and are not. necessary for conveying logical conjunction. There are no languages with zero disjunction because expressions with the function of or are semantically necessary (there is no operation of disjunction in the grammar), therefore they cannot be dispensed with if a language is to convey logical disjunction at all. The major cross-linguistic variations concerning the (non-)realization of conjunctive morphems are conceived as a result of morpho-syntactic processes that do not necessarily affect the semantic interpretation. Such processes are not directly related to the present discussion; these variations should be accounted for whatever semantic theory of coordination we adopt. To make things a bit more concrete, let me exemplify this line of reasoning using the following, somewhat trivial discourse:

A: Mary is walking. John is talking.

B: Is Mary walking (John talking)?

A: Sure, I've just told you: Mary is walking (John is talking).

B: So, Mary is walking and John is talking, ha?

A: Absolutely! Mary is walking and John is talking.

In this conversation we observe two facts:

Fact 1: $\quad(25) \Rightarrow(26) \quad$ Generally: $S_{1} S_{2} \Rightarrow S_{1}, S_{1} S_{2} \Rightarrow S_{2}$

Fact 2: $\quad(25) \Rightarrow(27) \quad$ Generally: $S_{1} S_{2} \Rightarrow S_{1}$ and $S_{2}$

Fact 2 is not that surprising given fact 1: if we accept the Boolean assumption we get $\llbracket S_{1}$ and $S_{2} \rrbracket=\llbracket S_{1} \rrbracket \wedge \llbracket S_{2} \rrbracket$. Due to fact 1 we have $S_{1} S_{2} \Rightarrow S_{1}, S_{1} S_{2} \Rightarrow S_{2}$. 
By the semantics of conjunction $\llbracket S_{1} \rrbracket, \llbracket S_{2} \rrbracket \Rightarrow \llbracket S_{1} \rrbracket \wedge \llbracket S_{2} \rrbracket$. We conclude that the entailment $S_{1} S_{2} \Rightarrow S_{1}$ and $S_{2}$ in fact 2 holds. A similar reasoning is possible with the universal of coordination since a conjunctive coordination always has the standard "intersective" reading like the one assigned by the Boolean assumption.

However, fact 1 is not explained by the Boolean assumption alone: in (25)-(26) there is no conjunctive coordinator, so why does the assertion of two consecutive sentences assert each of the sentneces separately? We must assume some morphologically null process of conjunction in discourse. This is what the univeral of coordination claims. But if such a process is to be assumed then there is no special virtue to the assumption that and conveys propositional conjunction: the conjunctive interpretation is available also if and is meaningless, using the free process. Therefore, if we want to account for fact 1 , we must assume a mechanism which makes the Boolean assumption dispensable for conjunction.

In cases of sentence internal conjunction, the universal of coordination makes similar predictions with respect to the cross-linguistic data. It claims that similarly to the free process of propositional conjunction in the interpretation of consecutive sentences in discourse, GC can apply also within the sentence in all languages, with no respect to the morphological presence of the conjunctive coordinator.

But how is the apparent zero disjunction as in (5) and (6) explained? As stated in $\left(\mathbf{G}_{1}\right)$, this question is empirically less central. Still, it is an interesting one. At least a partial answer comes from Gil's observation that the rare cases of zero disjunction appear with some expression signalizing uncertainty, like the suffix šaa in (5) and the clause I don't know in (6). It can be proposed that such expressions encode a semantic function of disjunction that applies to the structured meaning denoting the coordination and therefore blocks application of GC at this level. ${ }^{8}$ Surprisingly, cases where WS disjunction appears also involve some intensional particle. A possible approach to these cases is proposed in section 4.

\section{2 "Wide scope" conjunction}

More technically speaking, it is assumed above that the logic used to represent natural language sentences includes types with a product constructor $\bullet$, where an expression of type $\mathrm{a} \bullet \mathrm{b}$ is a tuple $\left\langle\varphi_{\mathrm{a}}, \psi_{\mathrm{b}}\right\rangle$ construed of the expressions $\varphi, \psi$ of types $\mathrm{a}$ and $\mathrm{b}$ by the axiom of product introduction. ${ }^{9}$ The natural deduction scheme and its corresponding semantics are as in van Benthem(1991:57):
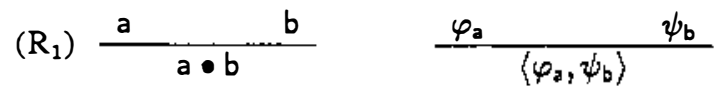

In product logics we have the equivalence $a(b c) \Leftrightarrow(a \bullet b) c$ (see van Benthem(1991:38,57)): any operator of type a(bc) can apply also to tuples of type $a \bullet b$ to produce a type c. Specifically, the operators GC and GD can apply to tuples- for any $t$-reducible type $\tau: \Pi_{\tau(\tau \tau)} \Leftrightarrow \Pi_{(\tau \bullet \tau) \tau}, \sqcup_{\tau(\tau \tau)} \Leftrightarrow \sqcup_{(\tau \bullet \tau) \tau}$.

The proposed interpretation of coordinations $X_{1}$ and $X_{2}$ in English and $X_{1}$ $\varnothing \mathrm{X}_{2}$ in languages with the zero strategy is the same compositional process. Given the denotations for $\mathrm{X}_{1}, \mathrm{X}_{2}$ the procedure consists of two stages:

(i) Interpreting the complex structure using the axiom $\left(R_{1}\right)$ : 


$$
\llbracket \mathrm{X}_{1} \text { andlø } \mathrm{X}_{2} \rrbracket=\llbracket \mathrm{X}_{1} \rrbracket \llbracket \text { and/ } \emptyset \rrbracket \llbracket \mathrm{X}_{2} \rrbracket=\llbracket \mathrm{X}_{1} \rrbracket \llbracket \mathrm{X}_{2} \rrbracket \stackrel{\mathrm{R}_{2}}{\Rightarrow}\left\langle\llbracket \mathrm{X}_{1} \rrbracket, \llbracket \mathrm{X}_{2} \rrbracket\right\rangle
$$

The coordinator and, like zero morphology, is analyzed as lacking any denotation.

(ii) An optional stage: applying the operator GC:

$$
\Pi\left\langle\llbracket \mathrm{X}_{1} \rrbracket, \llbracket \mathrm{X}_{2} \rrbracket\right\rangle \Rightarrow \llbracket \mathrm{X}_{1} \rrbracket \sqcap \llbracket \mathrm{X}_{2} \rrbracket \quad \text { (in short: }\left\langle\llbracket \mathrm{X}_{1} \rrbracket, \llbracket \mathrm{X}_{2} \rrbracket\right\rangle \stackrel{\mathrm{Gc}}{\Rightarrow} \llbracket \mathrm{X}_{1} \rrbracket \sqcap \llbracket \mathrm{X}_{2} \rrbracket \text { ). }
$$

Because (ii) is optional, the conjunctive coordination is ambiguous between the product reading and the intersective reading. A disjunction is not ambiguous in this way because the coordinator has the GD denotation so GC cannot apply:

$$
\llbracket \mathrm{X}_{1} \text { or } \mathrm{X}_{2} \rrbracket=\llbracket \mathrm{X}_{1} \rrbracket \llbracket \text { or } \rrbracket \llbracket \mathrm{X}_{2} \rrbracket=\llbracket \mathrm{X}_{1} \rrbracket \sqcup \llbracket \mathrm{X}_{2} \rrbracket
$$

To see how this mechanism works consider the following sentences:

(28) Every officer and gentleman danced.

(29) Every officer or gentleman danced.

Consider first the narrow scope derivation for the coordinations:

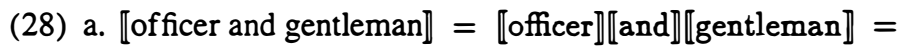

$$
\mathbf{o}_{\text {et }}^{\prime} \mathbf{g}_{\text {et }}^{\prime} \stackrel{\mathrm{R}_{1}}{\Rightarrow}\left\langle\mathbf{o}^{\prime}, \mathbf{g}^{\prime}\right\rangle \stackrel{\mathrm{GC}}{\Rightarrow} \mathbf{o}^{\prime} \sqcap \mathbf{g}^{\prime}
$$

$\llbracket$ every officer and gentleman danced $\rrbracket=\operatorname{every}_{(e t)((e t) t)}^{\prime}\left(o^{\prime} \sqcap g^{\prime}\right)\left(\right.$ dance $\left._{e t}^{\prime}\right)$

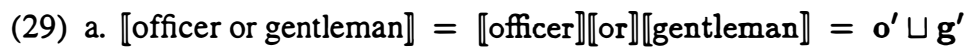

$\llbracket$ every officer or gentleman danced $\rrbracket=\operatorname{every}^{\prime}\left(o^{\prime} \sqcup \mathbf{g}^{\prime}\right)\left(\right.$ dance $\left.^{\prime}\right)$

In (28a) the optional operation of GC interprets the conjunction, which leads to a NS reading. In (29a) the disjunction is standardly interpreted as the NS reading. In order to get the WS reading of (28) consider the case where GC does not apply; we get the denotation of every applying directly to the tuple:

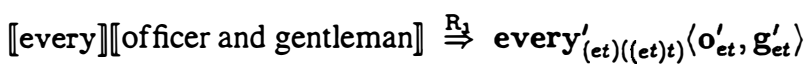

Traditionally, there is a type mismatch here: a function of type $(e t)((e t) t)$ cannot apply to a product of type $(e t) \bullet(e t)$. We will add a principle to the system to handle this kind of situation. Intuitively, we let the function every' apply pointwise to the coordinates in the product $\left\langle\mathbf{o}^{\prime}, \mathbf{g}^{\prime}\right\rangle$. This will yield the outcome $\left\langle\operatorname{every}^{\prime}\left(o^{\prime}\right)\right.$, every $\left.\left(g^{\prime}\right)\right\rangle$. This tuple can be interpreted using the free GC operation to get the WS representation: $\operatorname{every}^{\prime}\left(o^{\prime}\right) \sqcap$ every ${ }^{\prime}\left(g^{\prime}\right)$. To do this formally, we need a type shifting principle that turns a function $f$ (in the example above- every') of type ab into a function $\bar{f}$ of type $(a \bullet a)(b \bullet b)$ such that $\bar{f}\left(\left\langle x_{1}, x_{2}\right\rangle\right)=\left\langle f\left(x_{1}\right), f\left(x_{2}\right)\right\rangle$ for every $x_{1}, x_{2}$ of type a. This will be done using the following type schema:
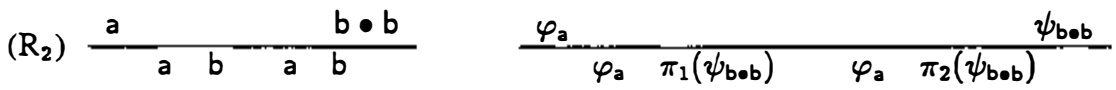

The left side is the type transition: whenever we have a type a with a product type $b \bullet b$ we can continue as if we have twice a type a with a type $b$. The right side is the semantics of this transition: $\pi_{1}(\psi)$ and $\pi_{2}(\psi)$ are the left and right coordinates of $\psi$, respectively, when $\psi$ is a tuple. These operators are undefined for non-tuples. The effect of this semantics is that whenever we have a $\lambda$-term $\varphi_{\mathrm{a}}$ together with a 
product type $\lambda$-term $\psi_{\mathrm{b} o b}=\left\langle\psi_{\mathrm{b}}^{1}, \psi_{\mathrm{b}}^{2}\right\rangle$, we can rewrite them using two occurrences of $\varphi_{\mathrm{a}}$, taking apart the tuple: $\varphi_{\mathrm{a}} \psi_{\mathrm{b}}^{1} \varphi_{\mathrm{a}} \psi_{\mathrm{b}}^{2}$. Let us see how $\left(\mathrm{R}_{2}\right)$ applies in the example above. Instantiate $\mathrm{a}=(e t)((e t) t), \mathrm{b}=e t, \varphi_{\mathrm{a}}=$ every $^{\prime}, \psi_{\mathrm{b} \text { ob }}=\left\langle\mathbf{o}^{\prime}, \mathbf{g}^{\prime}\right\rangle$ :

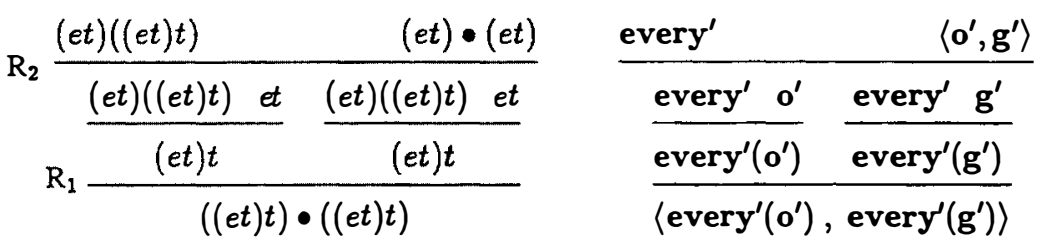

In short, this derivation means: every' $\left\langle\mathbf{o}^{\prime}, \mathbf{g}^{\prime}\right\rangle \Rightarrow\left\langle\operatorname{every}^{\prime}\left(\mathbf{o}^{\prime}\right)\right.$, every $\left.\mathbf{y}^{\prime}\left(\mathbf{g}^{\prime}\right)\right\rangle$ and using the free operation of GC: $\Rightarrow \operatorname{every}^{\prime}\left(o^{\prime}\right) \sqcap \operatorname{every}^{\prime}\left(g^{\prime}\right)$

Thus, the NP every officer and gentleman receives also an interpretation as a conjunction of quantifiers: $\left(\lambda P . \mathbf{o}^{\prime} \subseteq P\right) \Pi\left(\lambda P . \mathbf{g}^{\prime} \subseteq P\right)=\lambda P . \mathbf{o}^{\prime} \subseteq P \wedge \mathbf{g}^{\prime} \subseteq P$

and in (28): $\mathbf{o}^{\prime} \subseteq$ dance' $\wedge \mathbf{g}^{\prime} \subseteq$ dance', the WS reading of the conjunction. Note that with or a tuple of the conjuncts cannot be derived and therefore $\left(R_{2}\right)$ is blocked. Thus, WS disjunction is not predicted, in agreement with $\left(\mathbf{G}_{\mathbf{2}}\right)$.

The analysis of (13)-(18) is similar. However, the analysis of (7) depends on our treatment of transitive predicates and quantifier scope. The apparent WS disjunction in (19) also involves some problems of a larger scale. The discussion of these problems is deferred to section 4 .

\section{3 "Structure sensitive" adverbials}

In Lasersohn(1992:408) the following claim was made: "Structured Meanings provide the means for a straightforward and technically unproblematic account of alternately". I will support this claim by exemplifying the way in which the account given above for WS conjunction applies naturally also to sentences with adverbials like alternately and respectively. According to the line proposed, such adverbials denote functions that get a product type argument. The semantic operation can then apply using the separate denotations of the conjuncts, which are available in the product representation of the conjunction. Consider first the case of alternately in (21b), restated as (30) and paraphrased following Lasersohn as in (31):

(30) John was alternately hot and cold.

(31) There were at least two periods of time $\left[t_{1}, t_{2}\right]$ and $\left[t_{3}, t_{4}\right]\left(t_{1}<t_{2} \leq t_{3}<t_{4}\right)$ such that in $\left[t_{1}, t_{2}\right]$ John was hot and in $\left[t_{3}, t_{4}\right]$ John was cold. $t_{2}$ and $t_{3}$ should be sufficiently close $\left(t_{3}-t_{2}<c\right.$, for some small constant $\left.c\right)$.

Whether (31) is a completely adequate paraphrase of (30) is not our main concern here. What is crucial is the way the adverbial is sensitive to the separate denotations of the conjuncts. Assume $\llbracket$ hot $\rrbracket=$ hot $^{\prime}, \llbracket$ cold $\rrbracket=$ cold' denote functions from points of time to sets, i.e. they are of type $s(e t)$. Using the present account, altermately can be given a denotation which accepts a tuple of temporal predicates as an 
argument. The paraphrase in (31) leads to the following definition:

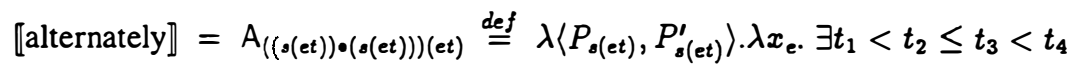

$$
\begin{aligned}
& {\left[t_{3}-t_{2}<c \wedge\left(\forall t\left(\left(t_{1} \leq t \leq t_{2} \rightarrow P(t)(x)\right) \wedge\left(t_{3} \leq t \leq t_{4} \rightarrow P^{\prime}(t)(x)\right)\right)\right)\right]^{10}}
\end{aligned}
$$

To simplify things, application of $A$ yields an et atemporal predicate, although in a temporal framework it should also be temporal. (30) is analyzed as follows:

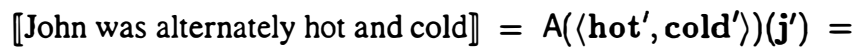

$$
\begin{aligned}
& \exists t_{1}<t_{2} \leq t_{3}<t_{4} \quad\left[t_{3}-t_{2}<c \wedge\right. \\
& \left.\left(\forall t\left(\left(t_{1} \leq t \leq t_{2} \rightarrow \operatorname{hot}^{\prime}(t)\left(\mathrm{j}^{\prime}\right)\right) \wedge\left(t_{3} \leq t \leq t_{4} \rightarrow \operatorname{cold}^{\prime}(t)\left(\mathrm{j}^{\prime}\right)\right)\right)\right)\right]
\end{aligned}
$$

In this analysis of (30) there is no use of GC. Here even an indirect relation between the conjunctive coordination and Boolean conjunction is unnecessary. Notice that NS conjunction using GC applying to the product denotation is excluded as it yields the denotation hot' $^{\prime} \Pi$ cold $^{\prime}$ of type $s(e t)$. This type can combine with the $((s(e t)) \bullet(s(e t)))(e t)$ type of the denotation of alternately using functional application only to derive $(s(e t))(e t)$, which leads to type mismatch in the following step (application to $\left.\llbracket \mathrm{John} \rrbracket=\mathrm{j}_{e}^{\prime}\right)$. Principle $\left(\mathrm{R}_{2}\right)$ cannot resolve this type mismatch since $A$ is not a product but a function over products. For similar considerations also disjunctive coordinations do not have the appropriate type as arguments for alternately. Thus, sentences like (22a-b) are semantically ill formed.

A similar treatment accounts for the semantics of respectively as in (23), restated:

(32) Mary and Sue are tall and short respectively.

The semantics given to the adverbial is the following:

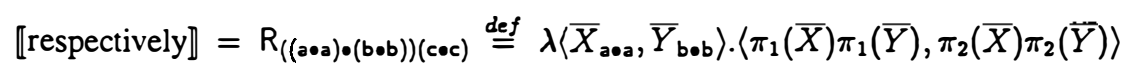

for all types $a, b, c$ s.t. $a, b \Rightarrow c$

In this definition respectively denotes a function $\mathrm{R}$ with two tuple arguments, $\vec{X}$ and $\bar{Y}$. The operation of $\mathrm{R}$ is to apply the first coordinates of $\bar{X}$ and $\bar{Y}$, and separately their second coordinates, to produce one tuple. GC can then apply to derive the meaning of the sentence. The analysis of (32) is as follows:

$\llbracket$ tall and short respectively $\rrbracket=\mathrm{R}\left(\left\langle\right.\right.$ tall $^{\prime}$, short $\left.\left.^{\prime}\right\rangle\right)=$ $\left[\lambda\left\langle\bar{X}_{(e t) \bullet(e t)}, \bar{Y}_{\text {eoee }}\right\rangle \cdot\left\langle\pi_{1}(\bar{X}) \pi_{1}(\bar{Y}), \pi_{2}(\bar{X}) \pi_{2}(\bar{Y})\right\rangle\right]\left(\left\langle\right.\right.$ tall $^{\prime}$, short $\left.\left.^{\prime}\right\rangle\right)=$ $\lambda \bar{Y}_{\text {eoe. }}\left\langle\left\langle\right.\right.$ tall $^{\prime} \pi_{1}(\bar{Y}) ;$ short $\left.^{\prime} \pi_{2}(\bar{Y})\right\rangle$

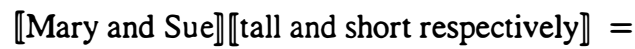
$\left[\lambda \bar{Y}_{\text {eoe }} \cdot\left\langle\operatorname{tall}^{\prime}\left(\pi_{1}(\bar{Y})\right), \operatorname{short}^{\prime}\left(\pi_{2}(\bar{Y})\right)\right]\left(\left\langle\mathbf{m}^{\prime}, \mathbf{s}^{\prime}\right\rangle\right)=\left\langle\operatorname{tall}^{\prime}\left(\mathbf{m}^{\prime}\right), \operatorname{short}^{\prime}\left(\mathbf{s}^{\prime}\right)\right\rangle \stackrel{\text { GC }}{\Rightarrow}\right.$ $\operatorname{tall}^{\prime}\left(\mathbf{m}^{\prime}\right) \wedge \operatorname{short}^{\prime}\left(\mathbf{s}^{\prime}\right)$

As in the case of altemately, when an or coordination is given as an argument to respectively type mismatch occurs, which eliminates sentences like (24). Consequently, the generalization in $\left(\mathbf{G}_{\mathbf{3}}\right)$ is accounted for.

Concluding, it seems that the proposed universal of coordination and its implementation using structured meanings provide a general account of the dis- 
tinctions between conjunctive and disjunctive coordinations. Some of the many problems that still need to be treated are discussed in the following section.

\section{Some broader theoretical issues}

\subsection{Quantifiers scope and flexibility}

Reconsider (7), restated here:

(33) John sold and bought a car. a. John sold a car and bought a car.

To derive the WS conjunction reading of (33), paraphrased in (33a), we should concentrate on the way the transitive predicate applies to the indefinite object. I will assume that the indefinite is standardly represented as a generalized quantifier of type (et)t. Let us consider three approaches to quantification in this situation:

(i) The Montague approach: The predicate has a quantifier type argument and a syntactic mechanism of Quantifying-in (Qin) allows WS quantifier readings. Extensionally, the predicate's type is then $((e t) t)(((e t) t) t)$.

(ii) The obligatory syntactic scope approach: assumed in much of the Logical Form literature and with semantic explicitness in Partee \& Rooth(1983) (henceforth $\mathrm{P} \& \mathrm{R})$. Transitive predicates get the $e(e t)$ type and object quantifiers must have syntactic scope using a mechanism like Quantifier Raising or Qin.

(iii) The flexible approach (see Hendriks(1993)): Transitive predicates are basically of type $e(e t)$. The scope of quantifiers is predicted by a semantic type lifting of the predicate, e.g. into type $((e t) t)(e t)$, which gets a quantifier argument.

It will be claimed that $\left(\mathbf{G}_{2}\right)$ shows an empirical advantage to the flexible approach using a "lazy" strategy of type lifting originating in P\&R. Consider first the Montague approach. As $P \& R(p .365)$ point out, this approach predicts that all transitive verb coordinations are interpreted as having a WS reading without any additional mechanism. As claimed in $\left(\mathbf{G}_{\mathbf{2}}\right)$, this is inadequate for or coordinations.

In the syntactic scope approach an opposite problem appears: even the mechanism proposed above cannot provide the WS conjunction reading of (33). The

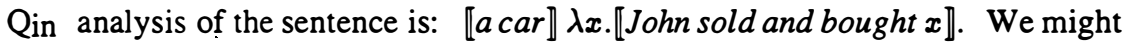
try to derive from this representation (using a principle like $\left(R_{2}\right)$ ) an equivalent to: $\llbracket a$ car $\rrbracket \lambda x$. $\llbracket J o h n$ sold $x$ and bought $x \rrbracket$. However, this still leads to a NS reading since both free occurrences of $x$ are bound by the quantifier denotation $\llbracket a$ car $\rrbracket$. A way to solve this in the scope approach is to assume a syntactic reconstruction of the object to get the paraphrase (33a). But according to $\left(\mathbf{G}_{2}\right)$, we have to explain why this process does not apply in or coordinations, which seems a hard task.

In the flexible approach to quantifier scope the picture changes a little. Simplifying many details of the mechanism in Hendriks(1993), the main operation for analyzing (33) is a lifting called Argument Raising (AR). In the case of $e(e t)$ predicates $\mathrm{AR}$ can be defined for the object argument as follows:

$$
\text { For every predicate } P_{e(e t)}: \quad \operatorname{AR}_{\mathrm{o}}(P) \stackrel{\text { def }}{=} \lambda Q_{(e t) t} \cdot \lambda x \cdot Q(\lambda y \cdot P(y)(x))
$$

This allows an (et)t quantifier argument to combine with an $A R_{0}$ lifted $e(e t)$ transitive predicate, yielding an intransitive et predicate. For example:

$\llbracket$ sell a car $\rrbracket=\left[A R_{o}\left(\operatorname{sell}_{e(e t)}^{\prime}\right)\right]\left(\lambda P_{e t} \cdot P \cap \operatorname{car}_{e t}^{\prime} \neq \phi\right)=$ 
$\left[\lambda Q . \lambda x \cdot Q\left(\lambda y \cdot \operatorname{sell}^{\prime}(y)(x)\right)\right]\left(\lambda P . P \cap \operatorname{car}^{\prime} \neq \phi\right)=\lambda x . \exists y\left(\operatorname{sell}^{\prime}(y)(x) \wedge \operatorname{car}^{\prime}(y)\right)$

In Hendriks's account the mechanism allows WS coordination equally with conjunction and disjunction due to the assumption that flexibility operators like $A R_{o}$ are freely allowed at any stage of the derivation. For example, we get WS disjunction:

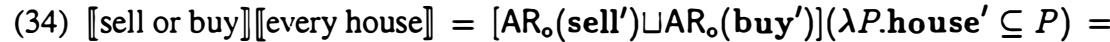
$\left[\left(\lambda Q \cdot \lambda x \cdot Q\left(\lambda y \cdot \operatorname{sell}^{\prime}(y)(x)\right)\right) \sqcup\left(\lambda Q \cdot \lambda x \cdot Q\left(\lambda y \cdot\right.\right.\right.$ buy $\left.\left.\left.^{\prime}(y)(x)\right)\right)\right]\left(\lambda P \cdot\right.$ house $\left.^{\prime} \subseteq P\right)=$ $\lambda x .\left[\forall y\left(\operatorname{house}^{\prime}(y) \rightarrow \operatorname{sell}^{\prime}(y)(x)\right) \vee \forall y\left(\operatorname{house}^{\prime}(y) \rightarrow \operatorname{buy}^{\prime}(y)(x)\right)\right]$

This disagrees with $\left(\mathbf{G}_{\mathbf{2}}\right)$. It might seem that the flexibility approach faces a similar problem to Montague's, deriving the undesired WS or reading. This is not necessarily the case. We readopt, instead of Hendriks's liberal approach to flexibility ("apply freely any type shifting available"), the "last resort" approach of P\&R: "apply type shifting only when functional application fails". In this approach the type lifting in (34) is unmotivated: $\mathrm{AR}_{\mathrm{o}}$ should not apply to the $e(e t)$ disjuncts because GD can apply directly without lifting. Lifting is required only when the verb predicate applies to the disjunction and type mismatch is detected. We get only:

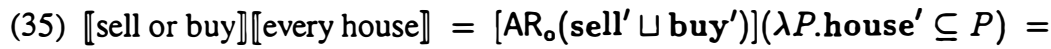
$\left[\lambda Q \cdot \lambda x \cdot\left(Q\left(\lambda y \cdot \operatorname{sell}^{\prime}(y)(x) \vee\right.\right.\right.$ buy $\left.\left.\left.^{\prime}(y)(x)\right)\right)\right]\left(\lambda P\right.$. house $\left.^{\prime} \subseteq P\right)=$ $\lambda x .\left[\forall y\left(\operatorname{house}^{\prime}(y) \rightarrow\left(\operatorname{sell}^{\prime}(y)(x) \vee\right.\right.\right.$ buy $\left.\left.\left.^{\prime}(y)(x)\right)\right)\right]$

(35) is the correct NS reading of the disjunctive predicate. However, for the same reason the lazy strategy with $A R_{0}$ alone does not derive the desired WS reading of the conjunction (33). Using the product mechanism proposed in section 3 it does:

$\llbracket$ sell and buy $\rrbracket \llbracket$ a car $\rrbracket=\left\langle\right.$ sell $^{\prime}$, buy $\left.^{\prime}\right\rangle \lambda P . P \cap$ car $^{\prime} \neq \phi \stackrel{\mathrm{R}_{2}}{\Rightarrow}$

sell $^{\prime} \quad \lambda P . P \cap$ car $^{\prime} \neq \dot{\phi}$ buy' $\lambda P . P \cap$ car $^{\prime} \neq \phi \stackrel{\text { AR }}{\Rightarrow}$

$\left[\mathrm{AR}_{\mathrm{o}}\left(\mathrm{sell}^{\prime}\right)\right]\left(\lambda P . P \cap \mathbf{c a r}^{\prime} \neq \phi\right)\left[\mathrm{AR}_{\mathrm{o}}\left(\mathrm{buy}^{\prime}\right)\right]\left(\lambda P . P \cap \mathbf{c a r}^{\prime} \neq \phi\right) \stackrel{\mathrm{R}_{1}}{\Rightarrow}$ $\left\langle\left[\mathrm{AR}_{\mathrm{o}}\left(\right.\right.\right.$ sell $\left.\left.^{\prime}\right)\right]\left(\lambda P . P \cap \mathrm{car}^{\prime} \neq \phi\right),\left[\mathrm{AR}_{\mathrm{o}}\left(\right.\right.$ buy $\left.\left.\left.^{\prime}\right)\right]\left(\lambda P . P \cap \operatorname{car}^{\prime} \neq \phi\right)\right\rangle \Rightarrow$ $\left\langle\lambda x . \exists y\left(\operatorname{sell}^{\prime}(y)(x) \wedge \operatorname{car}^{\prime}(x)\right), \lambda x . \exists y\left(\operatorname{buy}^{\prime}(y)(x) \wedge \operatorname{car}^{\prime}(x)\right)\right\rangle \stackrel{\text { GC }}{\Rightarrow}$ $\lambda x . \exists y\left(\operatorname{sell}^{\prime}(y)(x) \wedge \operatorname{car}^{\prime}(x)\right) \wedge \exists y\left(\operatorname{buy}^{\prime}(y)(x) \wedge \operatorname{car}^{\prime}(x)\right)$

Thus, lazy application of $A R_{o}$ with the product mechanism proposed can allow WS readings of transitive verb conjunction without deriving WS disjunction.

This is of course a very sketchy proposal for the interaction between quantifiers and conjunction scope. However, the crucial point is that $\left(\mathbf{G}_{\mathbf{2}}\right)$ provides evidence for a "lazy" implementation of Hendriks's operator. The somewhat radical view in Hendriks(1993) that expressions can have an infinite number of denotations is replaced by $P \& R$ 's more economical flexibility. Note that also the transitions $\left(R_{1}\right)$ and $\left(R_{2}\right)$ are employed in a lazy fashion: $\left(R_{1}\right)$ applies to conjuncts, which cannot functionally apply to each other; $\left(R_{2}\right)$ can be used to apply only when a tuple and another expression that cannot be interpreted using functional application are juxtaposed. Elsewhere ${ }^{11}$ I show how Partee \& Rooth's principle of "lazy flexibility" can be formally defined using Klein \& Sag(1985)'s Type Driven Translation.

\section{2 "Wide scope" or, attitudes and structured meanings}

Throughout this paper a constant effort is made to provide WS readings only for 
conjunctive coordinations while avoiding WS disjunction. As indicated above, this is not the common practice in the literature. It has been often assumed that the kind of phenomena exemplified by sentence (19), restated below, should be explained using the same mechanism that accounts for WS conjunction.

(36) John is looking for a cat or a dog.

a. John is looking for a cat or is looking for a dog.

Rooth \& Partee(1982) point out that (36) has a de dicto interpretation equivalent to (36a), not derived by the Montagovian treatment of intensionality. There are two possible conclusions: either some additional mechanism is responsible for the problematic reading, or something is wrong in Montague's possible world semantics for intensions. I will argue that: (i) If some additional mechanism is proposed to handle the WS of coordinations in intensional contexts, it is problematic to assume that it is the same mechanism responsible for WS conjunctions in extensional contexts. (ii) There is reason to think that the structured meanings approach to attitudes does not face the problem Montague's approach faces here. This suggests that (36) introduces a challenge for a theory of intensionality and not for a theory of coordination like the one proposed in this paper.

First, it seems that the problem generally occurs with coordination in the complement of mental attitude verbs. Consider the de dicto reading of (37):

(37) Mary says that John is going to marry Sue or Sue is going to divorce Bill.

a. Mary says something equivalent to "John is going to marry Sue or Sue is going to divorce Bill".

b. Mary says something equivalent to "John is going to marry Sue" or something equivalent to "Sue is going to divorce Bill".

If Mary says "Sue and John are going to get married" and does not actually speak of Sue and Bill it seems (37) is true in case the speaker is not sure what Mary says. (37a) is false in this situation and (37b) is true, so it seems the latter is a paraphrase of an available reading for (37). It is hard to compare (37) with a minimally different sentence with a conjunctive complement: if we replace in (37) the or with an and the revised paraphrases we get instead of (37a-b) become hardly distinguishable. However, with other attitude verbs the same tricky phenomenon seem to appear:

(38) Mary denies that John is going to marry Sue and Sue is going to divorce Bill. a. Mary says something falsifying the sentence "John is going to marry Sue and Sue is going to divorce Bill".

b. Mary says something falsifying the sentence "John is going to marry Sue" and something falsifying the sentence "Sue is going to divorce Bill".

Consider a situation in which Mary says: "I do not think John and Sue are going to get married but I'm sure Sue and Bill are going to divorce". It seems that in such a situation (38) can be interpreted as false, which attests reading (38b).

Independently, $\left(\mathbf{G}_{2}\right)$ shows motivation not to derive WS disjunction in extensional contexts. Moreover, while the "scope" of conjunction in extensional 
contexts seems to obey similar syntactic restrictions to the "scope" of quantification (see the following subsection), coordination in intensional contexts does not: in (37) and (38) the scope of the coordination appears over a that clause, unlike for example the scope of the quantifier in (39), which does not get the reading (39a):

(39) Mary says/denies that every student made noise.

a. * For every student Mary says/denies that he/she made noise.

In short, it seems that WS coordination in intensional contexts requires a different mechanism than the WS conjunction phenomena discussed in sections 2-3. The structured meanings approach to intensionality might shed some light on the problem. A crucial point in Cresswell(1985) is that an attitude to a proposition can be analyzed like any relation to an entity of type $e$. A propositional entity can have a fine structure, reflecting the structure of the sentence it stands for. A sentence like (40) can be modeled following a rough paraphrase as in (40a):

(40) Mary says that John is going to marry Sue.

a. Mary is in a relation of saying to a propositional entity representing the sentence "John is going to marry Sue".

For further details about this idea see Cresswell(1985). The central point for the present discussion is that there is no substantial difference in this approach between the predication in (40) and predication in extensional sentences like Mary meets John. In both cases the relation is between individuals, only in (40) one individual stands for a proposition. Thus, there is also no substantial difference between sentence (37) and a sentence like Mary meets John or Bill. But, in (37) the or coordination can be analyzed in two ways: (i) or in one disjunctive attitude. (ii) or in a disjunction between attitudes. Roughly, the analysis is as in (41a-b):

(41) a. Mary is in a relation of saying to the entity representing the disjunctive sentence "John is going to marry Sue or Sue is going to divorce Bill".

b. Mary is in a relation of saying to the a disjunction of theentities representing the sentences "John is going to marry Sue", "Sue is going to divorce Bill".

(41a) reflects the NS disjunction (within one attitude) in (37). (41b) reflects WS disjunction (between attitudes). (41b) should be derived from (37) standardly like any disjunction between entities (e.g. by lifting individuals to quantifiers and applying GD). If this intuitive proposal can be substantiated in a theory of mental attitudes, it might explain how or gets WS only in intensional contexts. Also, the structural restrictions on WS interpretation might turn out to be irrelevant since the cases of pseudo-WS over intensional verbs are a subcase of standard predication and do not involve any special scope mechanism.

\subsection{Syntactic island constraints and a possible synthesis}

The main concern so far was how to allow WS conjunction and "structure sensitive" modification. Nothing was said about possible constraints on the mechanism. As for the scope of quantification, it is widely accepted that syntactic islands that restrict overt extraction (e.g. of $w h$ phrases) govern also the scope of quantifiers. The null 
hypothesis (also expressed in Hendriks(1993:96)) is that the same constraints restrict also the scope of conjunction. A convenient case to check this is the Complex NP constraint. Compare (15), restated as (42), with (43):

(42) Mozart is easy to play for every pianist over 60 and below 20.

(43) ? Mozart is easy to play for every pianist who is over 60 and below 20.

The conjunction in (43), unlike (42), is within a syntactic island. This might explain why while (42) has the reasonable WS reading, (43) has only the absurd NS reading. A similar point holds with alternately modification. Contrast (44) with (45):

(44) John was alternately with people from France and from England.

a. John was alternately with people from France and with people from England. (45) ? John was alternately with people who came from France and from England. (46) John was with people fromFrance and from England.

These facts suggest that the connection in the product mechanism between WS conjunction and structure sensitive modification has an empirical motivation- both phenomena seem to be restricted by similar constraints. Moreover, sentence (44) shows a direct relation between the phenomenon of WS conjunction and alternately modification: (44) must be read as WS equivalent to (44a). If alternately is omitted, as in (46), the conjunction also has a NS reading, paraphrased as: "John was with people, each of them was both from France and from England". The mechanism proposed directly predicts this fact: as stressed in the analysis of (30), alternately cannot apply to a conjunction if GC applies to derive a NS reading.

\section{Conclusions}

Drawing a line between the semantics of conjunctive coordinations and the Boolean operator of con junction is not an easy theoretical move. In this paper I tried to show some central motivations for such a step. The way it was done was shown to lead to a more comprehensive semantic model of coordination.

\section{Endnotes}

- Thanks to Martin Everaert, Kai von Fintel, Danny Fox, Nissim Francez, Michael Moortgat, Bill Philip, Tanya Reinhart, Ivan Sag, Khalil Sima'an, Martijn Spaan, Henk Verkuyl, Carin Verspoor, Joost Zwarts and participants of SALT5 for remarks and discussions. Errors are exclusively mine. Special thanks to Martin Everaert, without his help this paper would not have been presented.

1 Throughout this paper I will not be explicit with respect to the specific mechanism that instantiates the types of the polymorphic denotation of the coordinator.

${ }^{2}$ Henceforth, I use the terms syncategorematic and categorematic in a purely semantic sense. Traditionally, the term syncategorematic conjunction would mean that the morpheme and is introduced by a rule like $\mathrm{X} \rightarrow \mathrm{X}_{1}$ and $\mathrm{X}_{2}$ and not by a lexical entry. Nothing in this paper implies this treatment. The schema for coordination might generally be $\mathrm{X} \rightarrow \mathrm{X}_{1} \mathrm{CX}_{2}$ where $\mathrm{C}$ is the coordinator category. But since the morpheme and is conceived as meaningless, some non-lexical semantic 
information is needed to interpret this rule when $\mathrm{C}=$ and. This semantic process is what is referred to here as syncategorematic conjunction.

${ }^{3} \varnothing$ indicates zero morphology, without any commitment to the syntactic analysis. 4 = juxtaposition of phrases. Thanks to Kai von Fintel for mentioning this to me.

5 Sentences of the same structure as (7) often do not get a WS reading without some pressure from our world knowledge. As claimed in Partee \& Rooth(1983), it is hard to observe any entailment from (ii) to (i):

(i) John caught and ate a fish. (ii) John caught a fish and ate a fish.

If John caught only one fish and ate another one (ii) is certainly true. Whether (i) can be asserted then is at least not clear. However, P\&R's general conclusion is problematic as sentences like (7) show, in agreement with Hendriks(1993:52).

- See Micheaux(1991) for a descriptive survey of similar facts in French. An idea which might have to do with this is the special treatment given to the indefinite article in the DRT tradition, where the indefinite article lacks any direct contribution to meaning. So, it is possible that in this treatment WS can be technically avoided in $\mathrm{CN}$ conjunctions. However, the mystery is even more interesting: with the generic interpretation for indefinites WS does seem to appear. Consider a variation on (15):

(i) Mozart is easy to play for a pianist over 60 years old and below 20 years old.

Conjunction scope in the generic (i) behaves like with the universal in (15).

7 A similar idea is seminally expressed in Gil(1991). Carlson(1983) discusses some problematic phenomena with the Latin morpheme que, but his conclusions are somewhat different. However, see the discussion of example (20).

8 An open question for this line is why cannot we have a disjunctive interpretation in a variation of (6) with an overt conjunctive morpheme:

(i) ? Who's coming? Mary is coming and John is coming... I don't know.

A possible answer is revising the approach in the spirit of Carlson(1983) to analyze and as a "marking morpheme" requiring application of GC at some possibly higher compositional level. However, this would leave cases of alternately...and... unexplained, since as we shall see below in these cases GC is unnecessary. I leave this question open, so the impossibility of (i) remains problematic.

- Standardly, the denotation $\llbracket\left\langle\varphi_{a}, \psi_{b}\right\rangle \rrbracket$ is in the Cartesian product of domains: $D_{\mathrm{a}} \times D_{\mathrm{b}}$. Since we are concerned mainly with the type transitions of products, this modeltheoretical aspect will not play a significant role in the analysis.

10 The notation $\lambda\left\langle X_{\tau}, Y_{\sigma}\right\rangle . f(X, Y)$ is only an abbreviation for the expression $\lambda \bar{Z}_{\tau \bullet \sigma} . f\left(\pi_{1}(\bar{Z}), \pi_{2}(\bar{Z})\right)$, which is equivalent to $\lambda X . \lambda Y . f(X, Y)$.

11 in a paper in progress: "Lazy flexibility in type calculus driven translation"

\section{References}

van Benthem, J.(1991), Language in Action, North-Holland.

Bergmann, M.(1982), "Cross-categorial semantics for conjoined common nouns", Linguistics and Philosophy 5: 399-401.

Carlson, G.(1983), "Marking constituents", in F. Henny and B. Richards(eds.), Linguistic Categories: Auxiliaries and Related Puzzles, vol. 1, Reidel. 
Carpenter, B.(1994), "Quantification and scoping: a deductive account", to appear in B. Carpenter, Lectures on Type Logical Semantics, MIT Press.

Cresswell, M.J.(1985), Structured Meanings, MIT Press.

Dixon, R.(1972), The Dyribal Language of North Queensland, Cambridge University Press, Cambridge.

Emms, M.(1993), "Some applications of categorial polymorphisms", ms. the CIS, München. DYANA deliverable.

Gil, D.(1991), "Aristotle goes to Arizona, and finds a language without and", in D. Zaefferer(ed.), Semantic Universals and Universal Semantics, Foris, Berlin.

Hendriks, H.(1993), Studied Flexibility, Ph.D. diss., University of Amsterdam.

Keenan, E., and L. Faltz(1985), Boolean Semantics for Natural Language, Reidel, Dordrecht.

Klein, E., and I. Sag(1985), “Type driven translation”, Linguistics and Philosophy 8: $163-201$.

Larson, R.(1985), "The syntax of disjunction scope", Natural Language and Linguistic Theory 3.

Lasersohn, P.(1992), "Generalized conjunction and temporal modification", Linguistics and Philosophy 15:381-410.

Micheaux, C.(1991), "Reducing the coordination of determiners: some principles", an internal report, University of Liège.

Moltmann, F.(1992), Coordination and Comparatives, Ph.D. diss., MIT.

Partee, B., and M. Rooth(1983), "Generalized conjunction and type ambiguity", in R. Bauerle et al.(eds.), Meaning, Use and Interpretation of Language, De Gruyter, Berlin.

Payne, J.(1985), "Complex phrases and complex sentences" in T. Shopen(ed.), Language Typology and Syntactic Description vol. 2, Complex Constructions, University Press, Cambridge.

Rooth, M., and B. Partee(1982), "Conjunction, type ambiguity and wide scope or", in Flickinger et al.(eds.), Proceedings of the First West Coast Conference on Fornal Linguistics, Stanford.

Winter, Y.(1994), "A unified semantic treatment of singular NP coordination", an unpublished ms., OTS, Utrecht University.

OTS, Utrecht University, Trans 10, 3512 JK Utrecht, The Netherlands

e-mail: yoad.winter@let.ruu.nl 